\title{
The "step feature" of suprathermal ion distributions: a discriminator between acceleration processes?
}

\author{
H. J. Fahr ${ }^{1}$ and H. Fichtner ${ }^{2}$ \\ ${ }^{1}$ Argelander Institut für Astronomie, Universität Bonn, Auf dem Hügel 71, 53121 Bonn, Germany \\ ${ }^{2}$ Institut für Theoretische Physik IV, Ruhr-Universität Bochum, 44780 Bochum, Germany \\ Correspondence to: H. Fichtner (hf@tp4.rub.de)
}

Received: 10 January 2012 - Revised: 2 July 2012 - Accepted: 27 July 2012 - Published: 5 September 2012

\begin{abstract}
The discussion of exactly which process is causing the preferred build-up of $v^{-5}$-power law tails of the velocity distribution of suprathermal particles in the solar wind is still ongoing. Criteria allowing one to discriminate between the various suggestions that have been made would be useful in order to clarify the physics behind these tails. With this study, we draw the attention to the so-called "step feature" of the velocity distributions and offer a criterion that allows one to distinguish between those scenarios that employ velocity diffusion, i.e. second-order Fermi processes, which are prime candidates in the present debate. With an analytical approximation to the self-consistently obtained velocity diffusion coefficient, we solve the transport equation for suprathermal particles. The numerical simulation reveals that this form of the diffusion coefficient naturally leads to the step feature of the velocity distributions. This finding favours - at least in regions of the appearance of the step feature (i.e. for heliocentric distances up to about $11 \mathrm{AU}$ and at lower energies) the standard velocity diffusion as a consequence of the particle's interactions with the plasma wave turbulence as opposed to that caused by velocity fluctuation-induced compressions and rarefactions.
\end{abstract}

Keywords. Space plasma physics (Transport processes)

\section{Introduction and motivation}

During recent years, an intense discussion has been led regarding the physical cause of the formation of suprathermal power law tails of pick-up ions (PUIs) in the solar wind. While various velocity powers do occur, there appears to be a preference for $v^{-5}$-tails (Fisk and Gloeckler, 2007)(see the examples shown in Fig. 1).
Such tails can, in principle, be explained by a number of basic processes, like efficient velocity diffusion due to interplanetary turbulence (e.g. Isenberg, 1987; Chalov et al., 1995; Fichtner et al., 1996; Isenberg, 2005), diffusive acceleration at interplanetary shocks (e.g. Baring and Summerlin, 2008) and multiple ion reflection resulting from shock surfing (e.g. Lee et al., 1996; Zank et al., 1996; le Roux et al., 2000) or newly discussed processes like the action of velocity fluctuation-induced compressions and rarefactions of the solar wind plasma (Fisk and Gloeckler, 2007; Zhang, 2010; Fahr and Siewert, 2011).

It appears, however, that a particular feature of many observations has not drawn any attention, so far, namely the step-like feature of the PUI velocity distributions (indicated in Fig. 1). It can be observed at least out to about $11 \mathrm{AU}$, as measurements with the New Horizons spacecraft revealed (McComas et al., 2010, see Fig. 3 therein). While this "step feature", as we will refer to it, has clearly been noticed (see, e.g., Fig. 1 in Fisk and Gloeckler, 2007), we are not aware that it has been explicitly addressed in detail from a theoretical perspective in the literature. A closer inspection of the step feature might, however, be valuable because it will help to answer the question of which of the various suggested processes is actually operating and causing this feature.

While the studies invoking diffusive acceleration at interplanetary shocks (Baring and Summerlin, 2008, Fig. 1 therein), shock drift during multiple reflections at a shock (le Roux et al., 2000, Fig. 3 therein), or the interaction of particles with 2-D turbulence (le Roux et al., 2001, who referred to the step feature as a "knee", Fig. 1 therein) could successfully generate a step-like feature, it is either rather less pronounced, demonstrated to exist at the solar wind termination shock only, or derived for a case without cooling, 


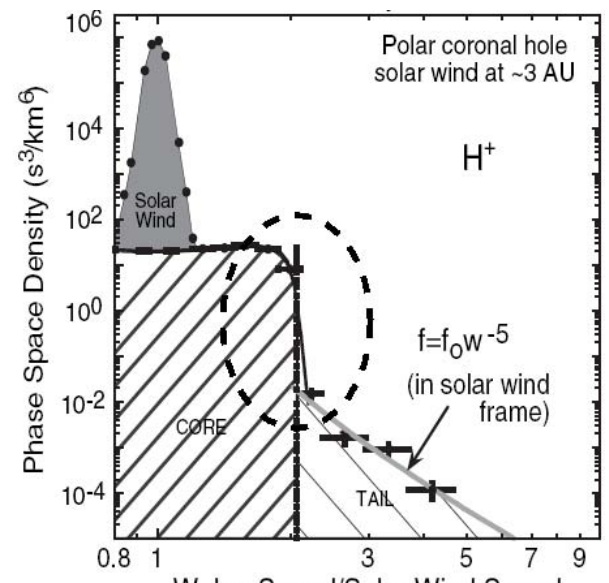

W Ion Speed/Solar Wind Speed

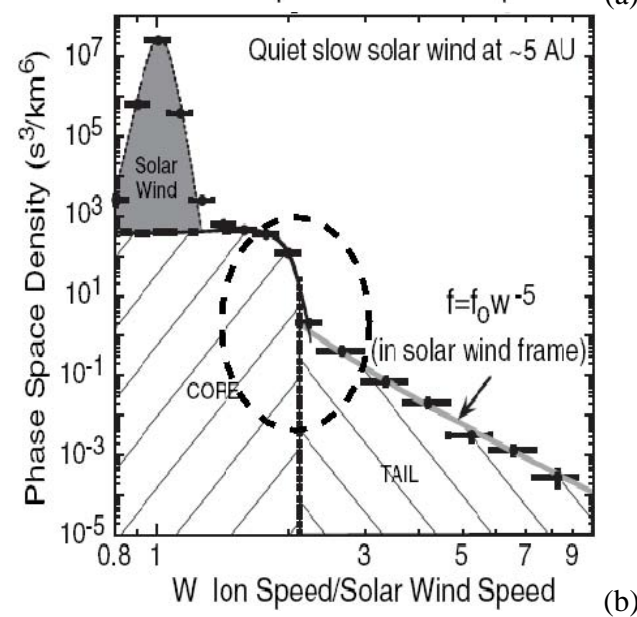

(b)

Fig. 1. The observed suprathermal ion tails in the fast (a) and slow (b) solar wind. Three populations are distinguished, namely the thermal solar wind as well as the suprathermal core and tail ions. The dashed lines encircle the step feature (adopted from Fisk and Gloeckler, 2007).

respectively. Schwadron et al. (1996) assumed transit-time damping in corotating interaction regions to be responsible for the tail formation, and could reproduce observations under the assumption of anisotropic turbulence, i.e. adopting a correlation length parallel to the local magnetic field 30 times longer than that perpendicular to the field.

Interestingly, two prime candidates (for a recent discussion, see Fahr and Fichtner (2011) and Fahr and Siewert (2011)) suggested to explain that the formation of extended tails appear to fail to reproduce the step feature: the models of velocity diffusion due to an interaction of PUIs with plasma waves (Isenberg, 2005) or with velocity fluctuations (Zhang, 2010) result in suprathermal tails reaching up to the level of the core distributions, i.e. in distributions exhibiting no step feature (see Figs. 7 and 2, respectively, in this paper).

We demonstrate here that standard velocity diffusion as originally suggested by Isenberg (1987) will also result in velocity distributions with a step feature if only a self- consistent treatment of the wave-particle interaction is taken into account. This fact favours this process as the one dominating at lower energies when compared to that suggested by Fisk and Gloeckler (2007). The effect of compressional fluctuations, suggested by them and critically assessed by Jokipii and Lee (2010), might take over in momentum diffusion efficiency at higher energies.

In the next Sect. 2, we derive a refined representation of the velocity diffusion coefficient resulting from self-consistent approaches; in Sect. 3 we describe and discuss the numerical simulation employing this coefficient, and in Sect. 4 we summarize the findings and draw corresponding conclusions.

\section{A diffusion threshold implied by a drop-off of velocity diffusion}

Several authors have studied the evolution of pick-up ions in a self-consistent approach regarding their interaction with plasma wave turbulence (see Bogdan et al. (1991), le Roux and Ptuskin (1998) and Chalov et al. (2004)). In these selfconsistent treatments, the velocity diffusion coefficient is represented by (see, e.g., Miller and Roberts, 1995)

$D_{v v}(r, v)=\frac{2 \pi^{2} e^{2}}{m^{2}}\left(\frac{v_{\mathrm{A}}}{c}\right)^{2} \frac{1}{v} \int_{k_{0}}^{\infty}\left[1-\left(\frac{k_{0}}{k}\right)^{2}\right] \frac{W(k)}{k} \mathrm{~d} k$

where $e, m$ and $v$ denote a particle's electric charge, mass, and speed, respectively, and $v_{\mathrm{A}}$ and $c$ are the Alfvén speed and the speed of light. The waves the particles interact with have the power spectrum $W(k)$ and are considered above a limiting wave number $k_{0}=\Omega / v$ with the particle's gyrofrequency $\Omega$.

The self-consistently computed wave spectra exhibit a cutoff at high wave number and can, in zeroth order be approximated by a constant $W(k)=W_{0}$ in the interval of interest (see Fig. 2). Consequently, the above integral can be simplified to

$D_{v v}(r, v)=D_{0}\left(\frac{U}{v}\right) \int_{k_{0}}^{k_{\max }}\left[1-\left(\frac{k_{0}}{k}\right)^{2}\right] \frac{1}{k} \mathrm{~d} k$

with $D_{0}=\left(2 \pi^{2} e^{2} v_{A}^{2} W_{0}\right) /\left(m^{2} c^{2}\right)$ (determined in magnitude by the PUI injection rate) and the solar wind speed $U$ used for normalization. Upon introducing the dimensionless variable $x=k / k_{0}$, it follows that 


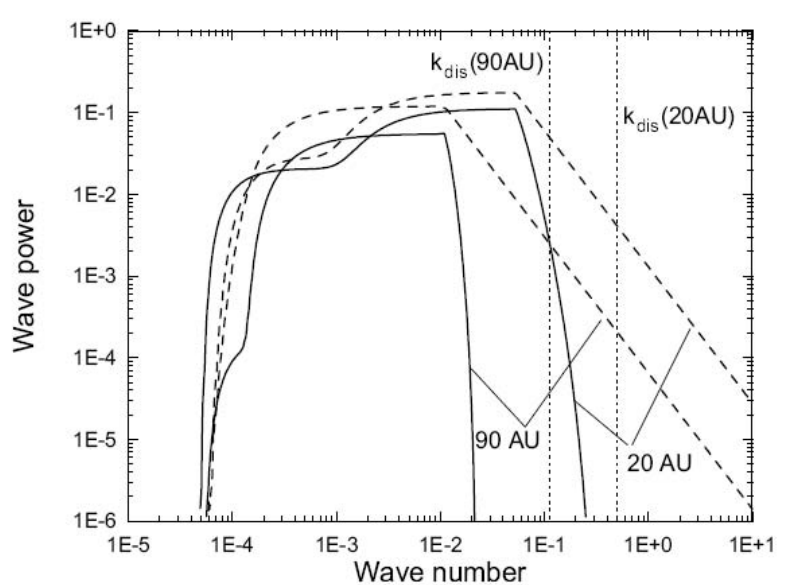

Fig. 2. The normalized wave power spectra $\tilde{W}(k)=$ $\left(e \sqrt{r_{0}} /(m c U) W(k)\right.$ as functions of the normalized wavenumber $\tilde{k}=k U / \Omega_{\mathrm{E}}$ as computed by Chalov et al. (2004). The solid lines are for the case that damping due to resonant interactions between the waves and pick-up protons is taken into account, while the dashed lines are spectral powers for vanishing damping. The vertical dotted lines show the solar wind proton dissipation wavenumbers.

$$
\begin{aligned}
D_{v v}(r, v) & =D_{0}\left(\frac{U}{v}\right) \int_{1}^{x_{\max }}\left[1-\frac{1}{x^{2}}\right] \frac{1}{x} \mathrm{~d} x \\
& =D_{0}\left(\frac{U}{v}\right) \int_{1}^{x_{\max }}\left[\frac{1}{x}-\frac{1}{x^{3}}\right] \mathrm{d} x \\
& =D_{0}\left(\frac{U}{v}\right)\left[\ln x+\frac{1}{2 x^{2}}\right]_{1}^{x_{\max }} \\
& =D_{0}\left(\frac{U}{v}\right)\left(\ln x_{\max }+\frac{1}{2 x_{\max }^{2}}-\frac{1}{2}\right)
\end{aligned}
$$

with

$x_{\max }=\frac{k_{\max }}{k_{0}} \approx \frac{\eta k_{\text {inj, } \mathrm{E}}}{k_{0}}=\eta\left(\frac{\Omega_{\mathrm{E}}}{U}\right)\left(\frac{v}{\Omega}\right) \approx\left(\frac{v}{U}\right)$

where, from Chalov et al. (2004), $\eta \Omega_{\mathrm{E}} / \Omega \approx 1$ is estimated. This approximation for the diffusion coefficient is plotted in Fig. 3.

Evidently, there is a threshold slightly above $v=U$, below which the velocity diffusion becomes inefficient. This threshold gives rises to a step feature in the velocity distribution of pick-up protons as is demonstrated in the next section.

\section{Numerical simulation}

Employing the same integration code as in Fahr and Fichtner (2011), we solved the standard transport equation for

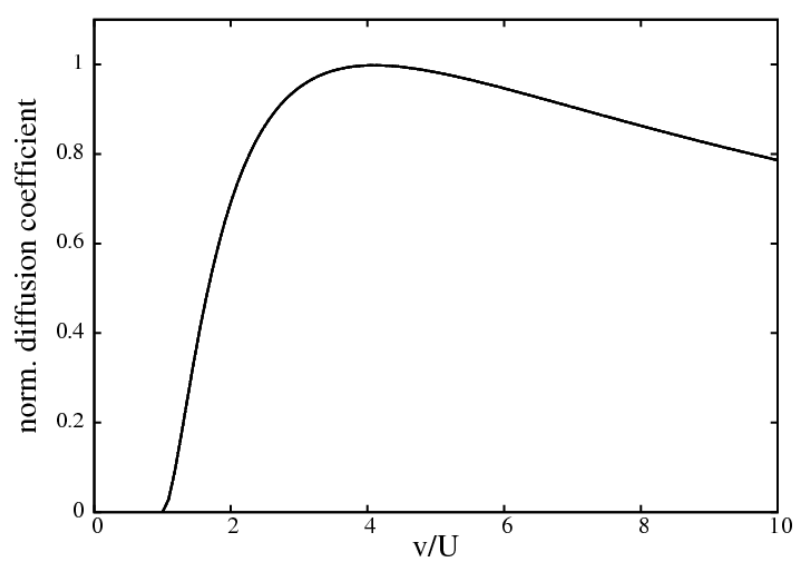

Fig. 3. The (normalized) approximated self-consistent velocity diffusion coefficient given by Eq. (3).

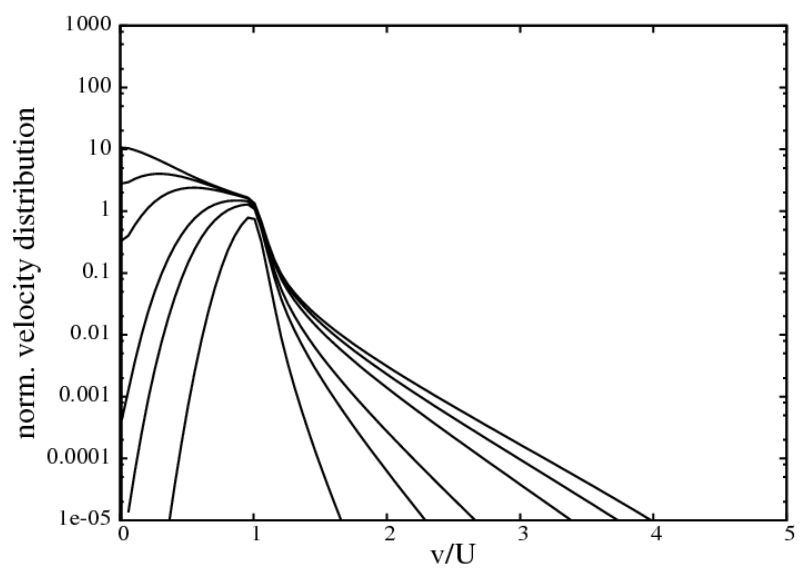

Fig. 4. The (normalized) PUI velocity distribution $f 2 U^{2} r / 3 Q$ as a function of normalized PUI speed and heliocentric distance (and, thus, of time): from bottom to top, the curves are for $r=1.2,1.6$, 2.0, 4.0, 8.0 and $29 \mathrm{AU}$ using the velocity diffusion coefficient given in Eq. (6) with the modification described with Eq. (3).

the omnidirectional, pitch angle-averaged velocity distribution function $f(r, v, t)$ :

$$
\frac{\partial f}{\partial t}=\frac{1}{v^{2}} \frac{\partial}{\partial v}\left(v^{2} D_{v v} \frac{\partial f}{\partial v}\right)+\frac{3 v U}{2 r} \frac{\partial f}{\partial v}-U \frac{\partial f}{\partial r}+S
$$

for the case of spherical symmetry. Here, $S=S(r, v, t)=$ $(Q / 2) \pi U^{2} H\left(t-t_{0}\right) \delta(v-U)$ is a source function with $Q=$ const. and $H$ denoting the Heaviside function.

For the simulation, we used the diffusion coefficient as in Fahr and Fichtner (2011) (see also Isenberg (1987)):

$D_{v v}(r, v)=$

$\frac{\pi v_{A 0}^{2} A_{0} U^{\gamma-3}}{\gamma(\gamma+2) B_{0}^{\gamma}}\left(\frac{q}{m c}\right)^{2-\gamma}\left(\frac{v}{U}\right)^{\gamma-1}\left(\frac{r}{r_{0}}\right)^{\gamma-\alpha}$

with $B_{0}=5 \times 10^{-5} \mathrm{G}, A_{0}=3.5 \times 10^{18} \mathrm{G}^{2} \mathrm{~cm}^{2}, \alpha=8 / 3$, and $v_{\mathrm{A}_{0}}=50 \mathrm{~km} \mathrm{~s}^{-1}$. Differently to our earlier simulations, 


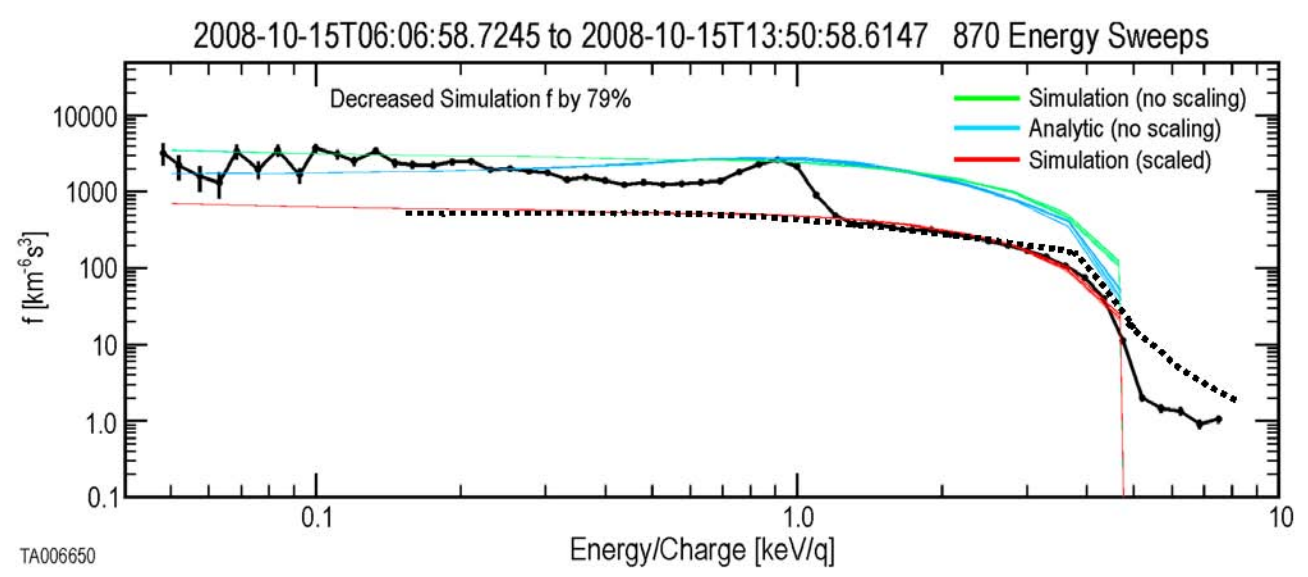

Fig. 5. The (PUI) distribution function as a function of energy per charge as measured with the New Horizon's spacecraft (black solid line; McComas et al., 2010). The blue line is an analytical approximation using the model by Vasyliunas and Siscoe (1976); the yellow/red line indicates the unscaled/scaled simulation result obtained by McComas et al. (2010), and the black dotted line is our (arbitrarily shifted) result (Figure adopted from McComas et al., 2010).

however, we consider here additionally the new velocitydependent factor from Eq. (3). For numerical reasons, the modification function was kept constant at 0.1 below $v / U=$ 1.2 .

This results in the velocity distributions displayed in Fig. 4. The strong decrease of the diffusion coefficient with decreasing particle speed makes diffusion efficient only a little above the injection speed. The cooling below and the acceleration above clearly result in the formation of the step feature.

That this step feature is indeed present out to at least $11 \mathrm{AU}$ can be seen from a comparison to the measurements with the New Horizon spacecraft (McComas et al., 2010) mentioned above: In Fig. 5, our results from Fig. 4 (here for $11 \mathrm{AU}$ ) are (after an arbitrary shift corresponding to a normalization constant) overplotted (as the black dotted line) on the figure published by McComas et al. (2010). Evidently, the step feature can clearly be identified in these data.

\section{Conclusions}

We have pointed out the significance of the so-called step feature in the total velocity distributions of the solar wind and suprathermal particles for a discrimination between processes that lead to the formation of power law tails.

We have demonstrated that standard velocity diffusion, as originally suggested by Isenberg (1987) and studied in some detail together with different cooling processes in Fahr and Fichtner (2011), will result in velocity distributions with a step feature if only a diffusion coefficient resulting from a self-consistent treatment of wave-particle interaction is taken into account. This finding - at least in regions of the appearance of the step feature, i.e. for heliocentric distances up to about $11 \mathrm{AU}$ and at low energies - favours this process over that suggested by Fisk and Gloeckler (2007), which has been critically assessed by Jokipii and Lee (2010) and studied in some more detail by Zhang (2010), Fahr and Siewert (2011), and Fahr et al. (2011), recently.

Acknowledgements. This research benefited from the meetings of the International Team devoted to understanding the $v^{-5}$-tails and ACRs that has been sponsored by the International Space Science Institute (ISSI) in Bern, Switzerland. We appreciate the discussions with Len Fisk, George Gloeckler, Nathan Schwadron and Vlad Izmodenov during these meetings.

Topical Editor M. Pinnock thanks R. Kallenbach and one anonymous referee for their help in evaluating this paper.

\section{References}

Baring, M. G. and Summerlin, E. J.: Particle Acceleration at Interplanetary Shocks, in: American Institute of Physics Conference Series, edited by: Li, G., Hu, Q., Verkhoglyadova, O., Zank, G. P., Lin, R. P., and Luhmann, J., vol. 1039 of American Institute of Physics Conference Series, 240-245, 2008.

Bogdan, T. J., Lee, M. A., and Schneider, P.: Coupled quasilinear wave damping and stochastic acceleration of pickup ions in the solar wind, J. Geophys. Res., 96, 161-178, doi:10.1029/90JA02096, 1991.

Chalov, S. V., Fahr, H. J., and Izmodenov, V.: Spectra of energized pick-up ions upstream. of the heliospheric termination shock I. The role of Alfvenic turbulences., Astron. Astrophys., 304, 609616, 1995.

Chalov, S. V., Alexashov, D. B., and Fahr, H. J.: Reabsorption of self-generated turbulent energy by pick-up protons in the outer heliosphere, Astron. Astrophys., 416, L31-L34, doi:10.1051/0004-6361:20040063, 2004.

Fahr, H. and Siewert, M.: Isotropic ion distribution functions triggered by consecutive solar wind bulk velocity jumps: 
a new equilibrium state, Astron. Astrophys., 527, A125+, doi:10.1051/0004-6361/201015619, 2011.

Fahr, H. J. and Fichtner, H.: Pick-up ion transport under conservation of particle invariants: how important are velocity diffusion and cooling processes?, Astron. Astrophys., 533, A92+, doi:10.1051/0004-6361/201116880, 2011.

Fahr, H. J., Chashei, I. V., and Siewert, M.: Solar wind bulk velocity fluctuations acting as velocity space diffusion on comoving ions, Astron. Astrophys., 537, A95+, doi:10.1051/00046361/201117503, 2012

Fichtner, H., Le Roux, J. A., Mall, U., and Rucinski, D.: On the transport of pick-up ions in the heliosphere, Astron. Astrophys., 314, 650-662, 1996.

Fisk, L. A. and Gloeckler, G.: Acceleration and Composition of Solar Wind Suprathermal Tails, Space Sci. Rev., 130, 153-160, doi:10.1007/s11214-007-9180-8, 2007.

Isenberg, P. A.: Evolution of interstellar pickup ions in the solar wind, J. Geophys. Res., 92, 1067-1073, doi:10.1029/JA092iA02p01067, 1987.

Isenberg, P. A.: Turbulence-driven Solar Wind Heating and Energization of Pickup Protons in the Outer Heliosphere, Astrophys. J., 623, 502-510, 2005.

Jokipii, J. R. and Lee, M. A.: Compression Acceleration in Astrophysical Plasmas and the Production of $\mathrm{f}(\mathrm{v})$ vprop $\mathrm{v}^{-5}$ Spectra in the Heliosphere, Astrophys. J., 713, 475-483, 2010.

le Roux, J. A. and Ptuskin, V. S.: Self-consistent stochastic preacceleration of interstellar pickup ions in the solar wind including the effects of wave coupling and damping, J. Geophys. Res., 103, 4799-4808, 1998. le Roux, J. A., Fichtner, H., and Zank, G. P.: Self-consistent acceleration of multiply reflected pickup ions at a quasi-perpendicular solar wind termination shock: a fluid approach, J. Geophys. Res., 105, 12557-12578, 2000.

le Roux, J. A., Matthaeus, W. H., and Zank, G. P.: Pickup ion acceleration by turbulent electric fields in the slow solar wind, Geophys. Res. Lett., 28, 3831-3834, 2001.

Lee, M. A., Shapiro, V. D., and Sagdeev, R. Z.: Pickup ion energization by shock surfing, J. Geophys. Res., 101, 4777-4790, 1996.

McComas, D. J., Elliott, H. A., and Schwadron, N. A.: Pickup hydrogen distributions in the solar wind at $\sim 11$ AU: Do we understand pickup ions in the outer heliosphere?, J. Geophys. Res.Space, 115, A03102, doi:10.1029/2009JA014604, 2010.

Miller, J. A. and Roberts, D. A.: Stochastic Proton Acceleration by Cascading Alfven Waves in Impulsive Solar Flares, Astrophys. J., 452, 912-932, 1995.

Schwadron, N. A., Fisk, L. A., and Gloeckler, G.: Statistical acceleration of interstellar pick-up ions in co-rotating interaction regions, Geophys. Res. Lett., 23, 2871-2874, 1996.

Vasyliunas, V. M. and Siscoe, G. L.: On the flux and the energy spectrum of interstellar ions in the solar system, J. Geophys. Res., 81, 1247-1252, 1976.

Zank, G. P., Matthaeus, W. H., and Smith, C. W.: Evolution of turbulent magnetic fluctuation power with heliospheric distance, J. Geophys. Res., 101, 17093-17107, 1996.

Zhang, M.: Acceleration of suprathermal particles by compressional plasma wave trains in the solar wind, J. Geophys. Res.Space, 115, A12102, doi:10.1029/2010JA015723, 2010. 